JOURNAL OF THE

CHUNGCHEONG MATHEMATICAL SOCIETY

Volume 27, No. 1, 2014

http://dx.doi.org/10.14403/jcms.2014.27.1.29

\title{
LORENTZIAN ALMOST $r$-PARA-CONTACT STRUCTURE IN TANGENT BUNDLE
}

\author{
Mohammad Nazrul Islam Khan* and Jae-Bok Jun**
}

\begin{abstract}
Almost contact and almost complex structures in the tangent bundle have been studied by K. Yano and S. Ishihara[5] and others. In the present paper, we have studied Lorentzian almost $r$ para-contact structure in the tangent bundle. Some results related to Lie-derivative have been studied.
\end{abstract}

\section{Introduction}

Let $M$ be a $n$-dimensional differentiable manifold of $C^{\infty}$ class and $T_{p}(M)$ the tangent space of $M$ at a point $p$ of $M$. Then the set $T(M)=$ $\bigcup_{p \in M} T_{p}(M)$ is called the tangent bundle over the manifold $M$. For any point $\tilde{p}$ of $T(M)$, the correspondence $\tilde{p} \rightarrow p$ determines the bundle projection $\pi: T(M) \rightarrow M$. Thus $\pi(\tilde{p})=p$, where $\pi: T(M) \rightarrow M$ defines the bundle projection of $T(M)$ over $M$. The set $\pi^{-1}(p)$ is called the fibre over $p \in M$ and $M$ the base space.

\section{Vertical lifts:}

If $f$ is a function in $M$, then we write $f^{V}$ for the function in $T(M)$ obtained by forming the composition of $\pi: T(M) \rightarrow M$ and $f: M \rightarrow R$ so that $f^{V}=f \circ \pi$. Thus, if a point $\tilde{p} \in \pi^{-1}(U)$ has induced coordinates $\left(x^{h}, y^{h}\right)$, then

$$
f^{V}(\tilde{p})=f^{V}(x, y)=f \circ \pi(\tilde{p})=f(p)=f(x) .
$$

Thus the value of $f^{V}(\tilde{p})$ is constant along each fibre $T_{p}(M)$ and equal to the value $f(p)$. We call $f^{V}$ the vertical lift of the function $f$.

Received August 21, 2013; Revised September 29, 2013; Accepted January 16, 2014 .

2010 Mathematics Subject Classification: Primary 53D15, 53B35.

Key words and phrases: tangent bundle, vertical lifts, complete lifts, Lie derivative.

Correspondence should be addressed to Jae-Bok Jun, jbjun@kookmin.ac.kr. 
Vertical lifts to a unique algebraic isomorphism of the tensor algebra $\Im(M)$ into the tensor algebra $\Im(T(M))$ with respect to constant coefficients by the conditions

$$
(P \otimes Q)^{V}=P^{V} \otimes Q^{V},(P+R)^{V}=P^{V}+R^{V},
$$

where $P, Q$ and $R$ are arbitrary elements of $\Im(M)$.

\section{Complete lifts:}

If $f$ is a function in $M$, then we write $f^{C}$ for the function in $T(M)$ defined by $f^{C}=i(d f)[4]$ and call $f^{C}$ the complete lift of the function $f$. The complete lift $f^{C}$ of a function $f$ has the local expression $f^{C}=$ $y^{i} \partial_{i} f=\partial f$ with respect to the induced coordinates in $T(M)$, where $\partial f$ denotes $y^{i} \partial_{i} f$.

Suppose that $X \in \Im_{0}^{1}(M)$. We define a vector field $X^{C}$ in $T(M)$ by $X^{C} f^{C}=(X f)^{C}$, where $f$ is an arbitrary function in $M$ and call $X^{C}$ the complete lift of $X$ in $T(M)$. The complete lift $X^{C}$ of $X$ with components $x^{h}$ in $M$ has components

$$
X^{C}=\left(\begin{array}{c}
x^{h} \\
\partial x^{h}
\end{array}\right)
$$

with respect to the induced coordinates in $T(M)$.

Suppose that $\omega \in \Im_{1}^{0}(M)$. Then a 1 -form $\omega^{C}$ in $T(M)$ is defined by $\omega^{C}\left(X^{C}\right)=(\omega(X))^{C}$, where $X$ is an arbitrary vector field in $M$. We call $\omega^{C}$ the complete lift of $\omega$.

The complete lifts to a unique algebra isomorphism of the tensor algebra $\Im(M)$ into the tensor algebra $\Im(T(M))$ with respect to constant coefficients is given by the conditions

$$
(P \otimes Q)^{C}=P^{C} \otimes Q^{V}+P^{V} \otimes Q^{C},(P+R)^{C}=P^{C}+R^{C},
$$

where $P, Q$ and $R$ are arbitrary elements of $\Im(M)$.

\section{Horizontal lifts:}

The horizontal lift $f^{H}$ of $f \in \Im_{0}^{0}(M)$ to the tangent bundle $T(M)$ is defined by $f^{H}=f^{C}-\nabla_{\gamma} f$, where $\nabla_{\gamma} f=\gamma(\nabla f)$.

Let $X \in \Im_{0}^{1}(M)$. Then the horizontal lift $X^{H}$ of $X$ is defined in $T(M)$ by

$$
X^{H}=X^{C}-\nabla_{\gamma} X
$$

where $\nabla_{\gamma} X=\gamma(\nabla X)$. The horizontal lift $X^{H}$ of $X$ has the components

$$
X^{H}=\left(\begin{array}{c}
x^{h} \\
-\Gamma_{i}^{h} x^{i}
\end{array}\right)
$$


with respect to the induced coordinates in $T(M)$, where $\Gamma_{i}^{h}=y^{j} \Gamma_{j i}^{h}$.

The horizontal lift $S^{H}$ of a tensor field $S$ of arbitrary type in $M$ to $T(M)$ is defined by

$$
S^{H}=S^{C}-\nabla_{\gamma} S .
$$

For any $P, Q \in \Im(M)$, we have

$$
\nabla_{\gamma}(P \otimes Q)=\left(\nabla_{\gamma} P\right) \otimes Q^{V}+P^{V} \otimes\left(\nabla_{\gamma} Q\right)
$$

or

$$
(P \otimes Q)^{H}=P^{H} \otimes Q^{V}+P^{V} \otimes Q^{H} .
$$

\section{Almost product structure}

Let $M$ be a $n$-dimensional differentiable manifold of $C^{\infty}$ class. If there exists a tensor field $F$ of type $(1,1)$ and of $C^{\infty}$ class on $M$ such that

$$
F^{2}=I,
$$

where $I$ denotes the unit tensor field. Then we say that $F$ gives to $M$ an almost product structure.

\section{Complete lifts of almost product structure and Lorentzian almost $r$-para-contact structure in the tangent bundle}

Let $\bar{M}$ be a $(2 n+r)$-dimensional differentiable manifold of $C^{\infty}$ class and $T(\bar{M})$ denotes the tangent bundle of $\bar{M}$. Suppose that there are given a tensor field $F$ of type $(1,1)$, a vector field $U_{\alpha}$ and a 1 -form $\omega^{\alpha}$ on $T(\bar{M})$ satisfying

$$
F^{2}=I+\sum_{\alpha=1}^{r} U_{\alpha} \otimes \omega^{\alpha},
$$

where

$$
\begin{aligned}
& F U_{\alpha}=0, \\
& \omega^{\alpha} \circ F=0, \\
& \omega^{\alpha}\left(U_{\beta}\right)=\delta_{\beta}^{\alpha} .
\end{aligned}
$$

Thus the manifold $\bar{M}$ satisfying conditions (3.1) and (3.2) will be said to possess Lorentzian almost $r$-para-contact structure([1], [3]). 
Theorem 3.1. Let $\bar{M}$ be a differentiable manifold endowed with Lorentzian almost $r$-para-contact structure $\left(F, U_{\alpha}, \omega^{\alpha}\right)$. Then

$$
\tilde{J}=F^{C}+\left(U_{\alpha}^{V} \otimes \omega^{\alpha V}-U_{\alpha}^{C} \otimes \omega^{\alpha C}\right)
$$

is an almost product structure on $T(\bar{M})$.

Proof. From (3.1) and (3.2), we have [2]

$$
\left(F^{C}\right)^{2}=I+\left(U_{\alpha}^{V} \otimes \omega^{\alpha C}-U_{\alpha}^{C} \otimes \omega^{\alpha V}\right)
$$

and

$$
\begin{aligned}
& F^{C} U_{\alpha}^{V}=0, F^{C} U_{\alpha}^{C}=0, \\
& \omega^{\alpha V} \circ F^{C}=0, \omega^{\alpha C} \circ F^{V}=0, \omega^{\alpha C} \circ F^{C}=0, \\
& \omega^{\alpha V}\left(U_{\alpha}^{V}\right)=0, \omega^{\alpha C}\left(U_{\alpha}^{C}\right)=1, \omega^{\alpha C}\left(U_{\alpha}^{V}\right)=1, \omega^{\alpha C}\left(U_{\alpha}^{C}\right)=0 .
\end{aligned}
$$

Let us define an element $\tilde{J}$ of $J(T(\bar{M}))$ by

$$
\tilde{J}=F^{C}+\left(U_{\alpha}^{V} \otimes \omega^{\alpha V}-U_{\alpha}^{C} \otimes \omega^{\alpha C}\right) .
$$

Then we find by (3.3), (3.4) and (3.5),

$$
\tilde{J}^{2}=I \text {. }
$$

Thus $\tilde{J}$ is an almost product structure in $T(\bar{M})$.

In view of equation (3.5), we have

$$
\begin{gathered}
\tilde{J} X^{V}=-(F X)^{V}+\left(\omega^{\alpha}(X)\right)^{V} U_{\alpha}^{C}, \\
\tilde{J} X^{C}=-(F X)^{C}-\left(\omega^{\alpha}(X)\right)^{V} U_{\alpha}^{C}-\left(\omega^{\alpha}(X)\right)^{C} U_{\alpha}^{C} .
\end{gathered}
$$

In particular, we have

$$
\begin{gathered}
\tilde{J} X^{V}=-(F X)^{V}, \tilde{J} X^{C}=-(F X)^{C}, \\
\tilde{J} U_{\alpha}^{V}=U_{\alpha}^{C}, \tilde{J} U_{\alpha}^{C}=U_{\alpha}^{C},
\end{gathered}
$$

where $X$ is an arbitrary vector field in $M$ such that $\omega^{\alpha}(X)=0$.

Theorem 3.2. Let the tangent bundle $T(M)$ of the manifold $M$ admits $\tilde{J}$ defined in (3.5). Then for any vector fields $X, Y$ such that $\omega^{\alpha}(Y)=0$, we have

(i) $\left(L_{X} V \tilde{J}\right) Y^{V}=0$,

(ii) $\left(L_{X} V \tilde{J}\right) Y^{C}=-\left(\left(L_{X} F\right) Y\right)^{V}+\left(\left(L_{X} \omega^{\alpha}\right) Y\right)^{V} U_{\alpha}^{C}$,

(iii) $\left(L_{X} V \tilde{J}\right) U_{\alpha}^{V}=\left(L_{X} U_{\alpha}\right)^{V}$,

(iv) $\left(L_{X} V \tilde{J}\right) U_{\alpha}^{C}=-\left(\left(L_{X} F\right) U_{\alpha}\right)^{V}+\left(L_{X} \omega^{\alpha}\left(U_{\alpha}\right)\right)^{V} U_{\alpha}^{C}$ 
and

(i)' $\left(L_{X} C \tilde{J}\right) Y^{V}=-\left(\left(L_{X} F\right) Y\right)^{V}+\left(L_{X} \omega^{\alpha}(Y)\right)^{V} U_{\alpha}^{C}$,

(ii)' $\left(L_{X} C \tilde{J}\right) Y^{C}=-\left(\left(L_{X} F\right) Y\right)^{C}-\left(\left(L_{X} \omega^{\alpha}\right) Y\right) U_{\alpha}+\left(\left(L_{X} \omega^{\alpha}\right) Y\right)^{C} U_{\alpha}^{C}$,

(iii)' $\left(L_{X} C \tilde{J}\right) U_{\alpha}^{V}=\left(\left(L_{X} F\right) U_{\alpha}\right)^{C}+\left[X, U_{\alpha}\right]^{C}+\left(\left(L_{X} \omega^{\alpha}\right) U_{\alpha}\right)^{V} U_{\alpha}^{C}$,

(iv)' $\left(L_{X} C \tilde{J}\right) U_{\alpha}^{C}=\left(\left(L_{X} F\right) U_{\alpha}\right)^{C}-\left[X, U_{\alpha}\right]^{V}-\left(L_{X} \omega^{\alpha}\left(U_{\alpha}\right)\right)^{V} U_{\alpha}$ $+\left(\left(L_{X} \omega^{\alpha}\right) U_{\alpha}\right)^{C} U_{\alpha}^{C}$

where $L$ is the Lie-derivative and [, ] is the Lie-Bracket.

Proof. The proof follows easily from (3.4), (3.6), (3.8) and [5].

4. Horizontal lifts of Lorentzian almost $r$-para-contact structure

Let $\left(F, U_{\alpha}, \omega^{\alpha}\right)$ be Lorentzian almost $r$-para-contact structure in $\bar{M}$ with an affine connection. Then we have form (3.1) and (3.2) and [5],

$$
\begin{gathered}
\left(F^{H}\right)^{2}=\left(I+U_{\alpha} \otimes \omega^{\alpha}\right)^{H}, \\
\left(F^{H}\right)^{2}=I+\left(U_{\alpha} \otimes \omega^{\alpha}\right)^{H}, \\
\left(F^{H}\right)^{2}=I+U_{\alpha}{ }^{H} \otimes \omega^{\alpha V}+U_{\alpha}{ }^{V} \otimes \omega^{\alpha H} .
\end{gathered}
$$

Also,

$$
\begin{gathered}
F^{H} U_{\alpha}^{H}=0, F^{H} U_{\alpha}^{V}=0, \\
\omega^{\alpha H}\left(U_{\alpha}^{H}\right)=0, \omega^{\alpha H}\left(U_{\alpha}^{V}\right)=1, \omega^{\alpha V}\left(U_{\alpha}^{H}\right)=1, \\
\omega^{\alpha H} \circ f^{H}=0, \omega^{\alpha V} \circ f^{H}=0 .
\end{gathered}
$$

Let us define a tenser field $\tilde{J}^{*}$ of type $(1,1)$ in $T(\bar{M})$ by

$$
\tilde{J}^{*}=F^{H}+U_{\alpha}^{V} \otimes \omega^{\alpha V}-U_{\alpha}^{H} \otimes \omega^{\alpha H} .
$$

Then it is easy to show from (4.1) and (4.2) that

$$
\tilde{J}^{* 2}=I,
$$

which means that $\tilde{J}^{*}$ is an almost product structure in $T(\bar{M})$. Thus we have

TheOREm 4.1. Let $\left(F, U_{\alpha}, \omega^{\alpha}\right)$ be Lorentzian almost $r$-para-contact structure in $\bar{M}$ with an affine connection $\nabla$. Then $\tilde{J}^{*}$ is a Lorentzian almost product structure in $T(\bar{M})$. 


\section{References}

[1] T. Adati, An almost $r$-para contact Riemanian manifold and an almost product Riemannian Manifold, Tensor, N. S. 43 (1986), 239-247.

[2] Lovejoy S. Das and Mohd. Nazrul Islam Khan, Almost r-contact structures on the tangent bundle, Differential Geometry-Dynamical Systems 7 (2005), 34-41.

[3] K. Matsumoto, On Lorentzian paracontact manifolds, Bull. of Yamagata Univ. Nat. Sci. 12 (1989), no. 2, 151-156.

[4] K. Yano and S. Ishihara, Almost complex structures induced in tangent bundles, Kodai Math. Sem. Rep. 19 (1967), 1-27.

[5] K. Yano and S. Ishihara, Tangent and cotangent Bundles, Marcel Dekker, Inc. New York, 1973.

Department of Computer Science

Qassim University Kingdom of Saudi Arabia

E-mail: mnazrul@rediffmail.com

$* *$

Department of Mathematics

Kookmin University

Seoul 136-702, Republic of Korea

E-mail: jbjun@kookmin.ac.kr 